Provided for non-commercial research and education use. Not for reproduction, distribution or commercial use.

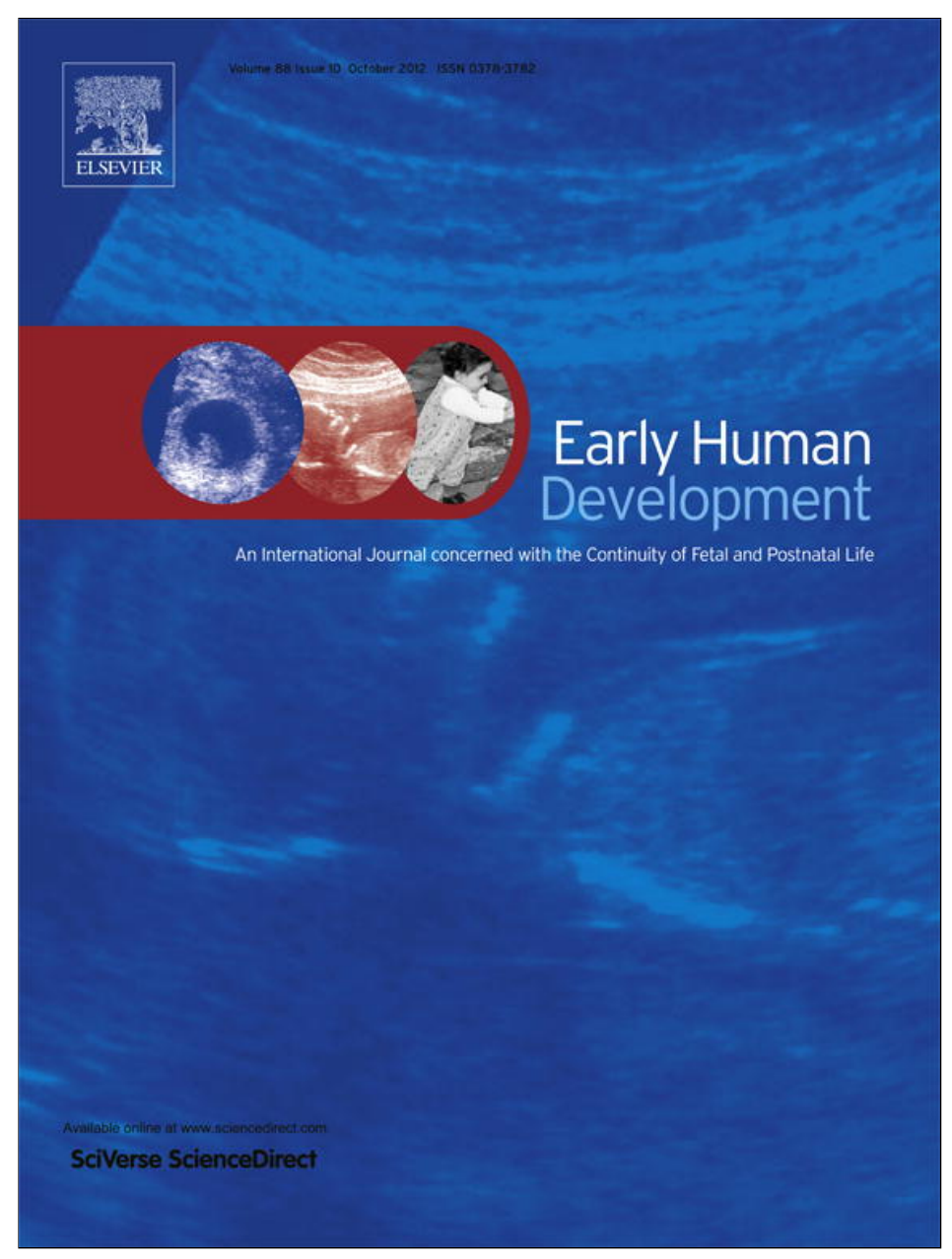

This article appeared in a journal published by Elsevier. The attached copy is furnished to the author for internal non-commercial research and education use, including for instruction at the authors institution and sharing with colleagues.

Other uses, including reproduction and distribution, or selling or licensing copies, or posting to personal, institutional or third party websites are prohibited.

In most cases authors are permitted to post their version of the article (e.g. in Word or Tex form) to their personal website or institutional repository. Authors requiring further information regarding Elsevier's archiving and manuscript policies are encouraged to visit:

http://www.elsevier.com/copyright 


\title{
Clinical and ultrasound features of placental maturation in pre-gestational diabetic pregnancy
}

\author{
Mary F. Higgins ${ }^{\text {a }}$, Noirin M. Russell ${ }^{\text {a }}$, Eoghan E. Mooney ${ }^{\mathrm{b}}$, Fionnuala M. McAuliffe ${ }^{\mathrm{a}, *}$ \\ a UCD Obstetrics and Gynaecology, School of Medicine and Medical Science, University College Dublin, National Maternity Hospital, Dublin, Ireland \\ b Department of Pathology, National Maternity Hospital, Dublin, Ireland
}

\section{A R T I C L E I N F O}

\section{Article history:}

Received 19 July 2011

Received in revised form 4 June 2012

Accepted 5 June 2012

\section{Keywords:}

Placenta

Diabetes

Pregnancy

Delayed villous maturation

\begin{abstract}
A B S T R A C T
Background: Pre-gestational diabetes (PGDM) is a significant cause of neonatal morbidity and mortality. Delayed villous maturation (DVM) is a placental diagnosis with increased risk of perinatal mortality.

Aims: This study aimed to prospectively look at the incidence of DVM in a PGDM population compared to non-diabetic controls. Additionally, we analysed antenatal ultrasound and clinical markers for DVM in the diabetic population.

Study design: This is a prospective study.

Subjects: Placentae of women with non-diabetic and PGDM pregnancies underwent detailed blinded histopathological examination.

Outcome measures: Clinical data, including birth weight, peri-natal outcome, and, in PGDM group, glycaemic control and ultrasound findings, were obtained.

Results: 77 non-diabetic women and 74 PGDM women consented to the study. The incidence of DVM in the PGDM group was higher than in the non-diabetic group (21/74 (28.4\%) vs. 11/77 (14.3\%) p=0.02; RR 1.98). In the PGDM group clinical and ultrasound markers were compared between the DVM group $(n=21)$ and the non-DVM group $(n=53)$. There was no difference in perinatal outcome nor glycaemic control between these two groups.

Conclusion: DVM, a placental finding with an increased risk of perinatal mortality, is increased in PGDM population compared to non-diabetic controls. No association was found with maternal glycaemic control. The presence of placental DVM was not associated with antenatal ultrasound parameters nor clinical perinatal outcome.
\end{abstract}

(c) 2012 Elsevier Ireland Ltd. All rights reserved.

\section{Introduction}

Pre-gestational diabetes mellitus complicates less than $1 \%$ of all pregnancies but is a significant cause of fetal morbidity and mortality [1]. Classically, chronic hypoxia, hyperglycaemia and lactic acidosis are thought to contribute to the risk of intrauterine death but placental function may also play a part. Even well controlled pre-gestational diabetic mothers with normally grown fetuses have an increased risk of perinatal mortality and morbidity, including fetal cardiomyopathy and late third trimester unexplained fetal death [2-5].

The placenta is the essential link between mother and fetus. Placental functions, which are essential for growth and development of the fetus, include gaseous exchange, provision of nutrients, hormonal excretion and transmission of maternal antibodies [6,7]. Abnormalities of implantation and intrinsic diseases of the placenta result in many of

\footnotetext{
* Corresponding author. Tel./fax: + 3531 6373216x6627586

E-mail address: Fionnuala.mcauliffe@ucd.ie (F.M. McAuliffe).
}

the common obstetric pathologies including pre-eclampsia, intrauterine growth restriction, fetal hypoxia, and sudden intrauterine death [8].

Delayed villous maturation (DVM) is a placental finding that is increased in maternal pre-gestational diabetes mellitus and is associated with an increased rate of perinatal mortality [8-12]. As such DVM may be one of the links between maternal diabetes and increased risk of intrauterine death in the fetus.

There is little published in the literature about DVM. One of the earliest studies showing an association between diabetes and villous maturation was that of Thomsen and Lieschke in 1958 [13]; further studies by Vogel [14] and Fox [15] supported the association between maternal diabetes and villous immaturity. The significance of this association was uncertain until one study [13] reviewing placental findings associated with stillbirth showed an incidence of "placental defect" (their term for DVM) of $52 \%$; of these $11 \%$ had a maternal history of diabetes.

A few studies have investigated the association between the incidence of DVM and type of diabetes (based on White classification). One study showed an increased risk of DVM in Class B and C diabetics compared to non-diabetic controls [16]; however a second study 
showed that the incidence of DVM in Whites Class D diabetic placentae was similar to those of non-diabetic placentae [17]. This discrepancy may be supported by the hypothesis that diabetic vasculopathy, more common in Type D diabetes, may result in placental over growth as a result of relative accelerated villous maturity. Another study investigated possible differences between placental findings of average weight and large for gestational weight infants of diabetic mothers and showed no difference in the incidence of either moderate or severe villous immaturity between the two groups [18]. Finally, Laurini et al. investigated incidence of DVM in 22 women whose diabetes was treated with continuous sub cutaneous insulin [19]. Despite near normal glycaemia amongst the diabetic women the incidence of villous immaturity was increased compared to non-diabetic controls; this, when extensive, was related to antenatal fetal asphyxia. They suggested that an increase in diffusion distance in the villi as a result of DVM could result in chronic fetal hypoxaemia. Haemoglobin oxygen affinity is increased in diabetic women compared to non-diabetic controls in late pregnancy [20] and this increased affinity is directly proportional to increases in HbA1c. Therefore it is possible that poorer glycaemic control would both increase the incidence of DVM and worsen the effects of it.

These studies to date have involved relatively small numbers. In addition, to our knowledge no study to date has related DVM to ultrasound measurements and clinical features, which may allow antenatal prediction of DVM. The aim, therefore, of this study was to investigate prospectively the incidence of DVM in non-diabetic low risk pregnancies and to compare it to the incidence in women with pre-gestational diabetes. Clinical and ultrasound parameters in women with pre-gestational diabetes, that might predict those who would develop placental DVM, were also investigated.

\section{Research design and methods}

The National Maternity Hospital is a tertiary level unit, one of three serving the city of Dublin and its environs. Over 9000 women deliver infants greater than 24 week gestation per year in the unit. The Diabetic Clinic in the National Maternity Hospital is one of the largest in Ireland, caring for $40-50$ women per year with pregestational diabetes and over 150 women with gestational diabetes. Women with pre-gestational diabetes normally attend from early pregnancy (5-6 weeks' gestation), and attend the clinic fortnightly throughout pregnancy. Those requiring oral hypoglycaemic medication prior to pregnancy are routinely converted to insulin therapy in early pregnancy. Ultrasound assessment was performed in the first (for dating and viability) second trimester (fetal anatomy including echocardiography) and regularly in the third trimester for fetal well being and growth. Unless spontaneous labour occurs, delivery is indicated for women with pre-gestational diabetes at 38 weeks if macrosomia is suspected or if the mother has poor glycaemic control; otherwise delivery is planned at $39-40$ week gestation.

With institutional ethics approval from January 2006 to January 2008 women with pre-gestational diabetes (PGDM) were invited to participate in the first trimester in a prospective research study investigating placental function in diabetic pregnancy. Women without PGDM who screened negative for gestational diabetes were invited to participate in the third trimester and these served as normal controls. Placentae from both groups underwent histological examination by one operator (EEM). Participants and their babies were followed up for six week postnatally.

Our method of histological placental examination has been outlined elsewhere [8]. Briefly, the cord and membranes were examined and removed. Placental weight and dimensions were recorded, and whether the specimen was weighed fresh or fixed was noted. One block of cord and membranes and five blocks of full-thickness parenchyma from the central $2 / 3$ of the disk were processed and 5 micron sections stained with haematoxylin and eosin. Cases were examined by an experienced placental pathologist who was blinded to the glycaemic status of the patient: other details such as gestational age were provided.

DVM represents a spectrum of disease varying from mild to severe with characteristic features being of tertiary villi with decreased vasculosyncytial membranes, a continuous cuboidal trophoblast covering and increased villous stroma compared to normal (Fig. 1). In the more severe grades there are decreased numbers of tertiary villi overall and those villi which are present are increased in volume and may be bulbous $[8,11]$. DVM may also be called villous immaturity or villous dysmaturity but all share a common finding in that tertiary villi are less mature than would be expected for the gestational age of the placenta. Any other pathology present was noted. Chorioamnionitis was staged as 1 to 4 [21]. Villitis was graded as 1-4 [22].

Clinical data on both normal and PGDM pregnancies was obtained from a variety of sources including the labour ward database, ultrasound department database and patient records. Participant characteristics such as maternal age, parity, gestational age at delivery and type of delivery were recorded as well as infant outcomes such as birth weight, birth weight centile, Apgars at 1 and 5 min and any reason for admission to the neonatal intensive care unit (NICU). Gestation was defined clinically as number of completed weeks since date of certain last menstrual period or by ultrasound measurement if dates were uncertain. Birth weight was weight following delivery as measured by a paediatrician or midwife using standardised scales (Seca 1 Model 335, Vogel and Halke, Germany). The gestation and gender appropriate centile was determined using the growth charts (Child Growth Foundation, London 1996) used in this institution. Poor perinatal outcome was defined as an arterial cord $\mathrm{pH}$ less than 7.2, or Apgars at 5 min less than 7 , or admission to the neonatal intensive care unit for an indication other than hypoglycaemia. Pregnancy outcomes for the women with diabetes further included measures of glycaemic control such as HbA1c and fructosamine throughout pregnancy, as well as White classification of diabetes. In the White classification, Class B is onset of diabetes at age 20 or older or with duration of less than 10 years; Class C: onset at age $10-19$ or duration of $10-19$ years and Class D: onset before age 10 or duration greater than 20 years. Class $\mathrm{N}$ comprises all those with diabetic nephropathy and Class $\mathrm{R}$ all those with diabetic retinopathy.

In addition, women with PGDM underwent the routine third trimester ultrasound examinations, assessing fetal wellbeing and fetal growth. These examinations were performed at 30, 33 and 36 week gestation and then weekly until delivery. At each examination fetal growth (as measured by bi-parietal diameter, head circumference, abdominal circumference and femur length), fetal wellbeing (biophysical profile) and Doppler assessment (of the umbilical artery and middle cerebral artery) was performed. Ultrasound measurements were performed by one of three trained operators (MH, NR, FMcA). All examinations were performed transabdominally using either a Toshiba Xario (Toshiba Medical Systems Corporation, Japan) or Voluson 730 Expert, (GE Medical Systems, Germany) equipped with curved array transducers.

\subsection{Statistical analysis}

Statistical analysis was performed using SPSS version 12 (SPSS Inc, Chicago, IL). Data was assessed for normality using Shapiro Wilk and P-P plot; normally distributed data was compared using $t$-test (means $+/-$ standard error). Non-parametric data was compared using Mann-Whitney test (median/range). Binomial data was assessed using the chi-squared test for proportions or Fisher's exact test. Multiple linear analyses using forward stepwise analysis were performed. Statistical significance was set at $\mathrm{p}<0.05$.

\section{Results}

From January 2006 to March 2008151 women consented to take part in the study. Of these 77 were women with low-risk, normal, 

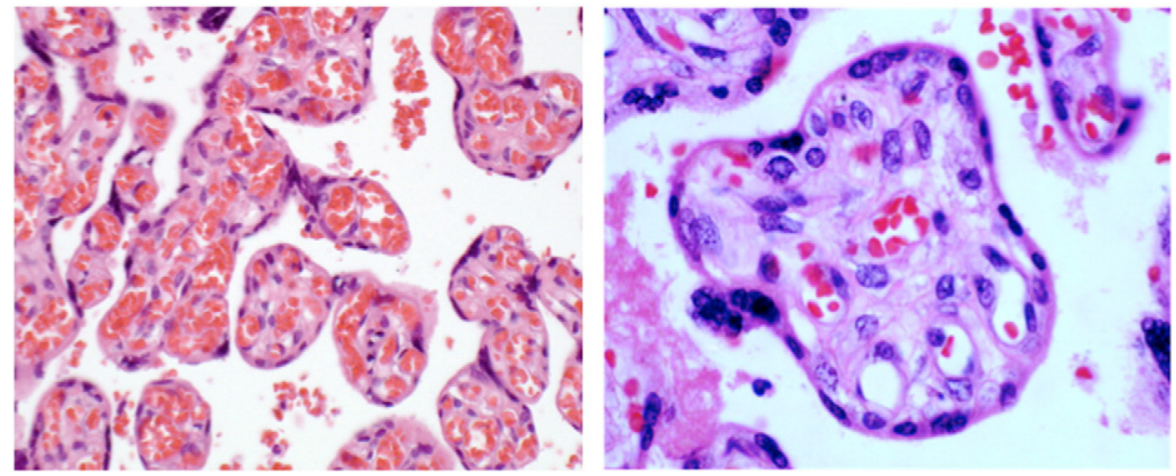

Fig. 1. Delayed villous maturity. Mild (left), moderate (middle column) and severe (right column) are shown under low power (200×, upper row) and high power (400×, lower row). There is a progressive increase in stromal volume, with decreased relative capillary volume and increased diffusion distance from mild to severe forms of DVM.

non-diabetic singleton pregnancies and 74 were women with PGDM with singleton pregnancies. Background characteristics of both groups are shown in Table 1. There were no intra-uterine or neonatal deaths in any of the participants during this time; follow up for neonatal deaths was until the six-week postnatally.

Within the group of women with PGDM nine women had a diagnosis of type 2 diabetes mellitus; all required oral hypo-glycaemics to maintain normoglycaemia prior to pregnancy and were converted to subcutaneous insulin on booking in early pregnancy. The remainder of women had type 1 diabetes mellitus with the majority $(46 / 74,62 \%)$ being Whites class $\mathrm{B} / \mathrm{C}$; three women were Class $\mathrm{R}$ (retinopathy) and the remainder was class D; there were no women with diabetic nephropathy in the study.

Overall mean (SD) HbA1c in the pre-gestational diabetic group was as follows: early pregnancy $7.4 \%(+/-1.3 \%), 14$ week gestation $6.4 \%(+/-0.7 \%), 20$ week gestation $(6.1 \%+/-0.7 \%)$, and at 36 week gestation $6.2 \%(+/-0.8 \%)$.

Amongst the women with PGDM the incidence of a placental diagnosis of DVM was $28.4 \%$ (21/74) compared to an incidence of DVM in the control group of $14.3 \%$ (11/77) (chi squared test for proportions, $\mathrm{p}=0.02$ ). Thus the incidence of DVM in the PGDM cohort is nearly double that of controls (RR 1.98; 95\% Cl 1.03-3.74). Other histopathological outcomes are shown in Table 2.

Multiple linear regression was performed looking at prediction of placental DVM, including possible confounders such as maternal age,

Table 1

Clinical characteristics of participants: non diabetic normal controls and women with pre-gestational diabetes mellitus.

\begin{tabular}{llll}
\hline & $\begin{array}{l}\text { Non diabetic normal } \\
\text { controls }(\mathrm{n}=77)\end{array}$ & $\begin{array}{l}\text { Pre-gestational diabetes } \\
\text { mellitus }(\mathrm{n}=74)\end{array}$ & $\begin{array}{l}\mathrm{p} \\
\text { value }\end{array}$ \\
\hline $\begin{array}{l}\text { Maternal age (years) } \\
\text { Parity (\% primiparous) }\end{array}$ & $31(+/-0.5)$ & $33(+/-0.5)$ & 0.04 \\
$\begin{array}{l}\text { Gestational age at } \\
\quad \text { delivery (weeks + days) }\end{array}$ & $39+1(+/-1$ day) & $31 / 74$ & 0.51 \\
Birth weight (g) & $3440(2140-4950)$ & $3650(2635-4875)$ & 0.04 \\
Birth weight centile & $50(2-99)$ & $91(2-99)$ & $<0.01$ \\
Placental weight $(\mathrm{g})$ & $466(252-878)$ & $513(305-882)$ & $<0.01$ \\
Placental volume $\left(\mathrm{cc}^{3}\right)$ & $444(240-836)$ & $487(290-840)$ & 0.04 \\
Apgars $<7$ at 1 min & 0 & 0 & - \\
Apgars $<7$ at 5 min & 0 & 0 & - \\
Type of delivery & & & $<0.01$ \\
$\quad$ Normal vaginal & $42 / 77(54 \%)$ & $29 / 74(39 \%)$ & $<0.01$ \\
$\quad$ Instrumental & $14 / 77(18 \%)$ & $7 / 74(9 \%)$ & $<0.01$ \\
$\quad$ LSCS & $21 / 77(27 \%)$ & $38 / 74(51 \%)$ & $<0.01$ \\
Gender (\% female) & $40 / 77(52 \%)$ & $55 / 74(74 \%)$ & $<0.01$ \\
Admission to NICU (\%) & $2 / 77(2 \%)$ & $26 / 74(35 \%)$ & \\
\hline
\end{tabular}

NICU neontatal intensive care unit. Maternal age (in years), gestational age (in weeks + days from first day of last menstrual period/assigned from dating ultrasound) at delivery are all reported as mean ( $+/-$ standard error). Birth weight (in grammes) and birth weight given as median (range). maternal BMI, gestational age at delivery, gender of infant as well as presence of maternal diabetes. Maternal diabetes was the most significant factor $(r=0.23, p=0.01)$ with gender of infant being the last to be excluded $(p=0.1)$ (maternal age $(p=0.26)$, gestational age $(p=0.48)$, maternal BMI $(p=0.82))$. There was no difference between women with type 1 diabetes and type 2 diabetes, though this is limited due to the small number of women in the study with type 2 diabetes $(n=9)$.

Ultrasound and clinical parameters were compared in the diabetic population alone, comparing ultrasound measurements, glycaemic control and length of diabetes in PGDM women with and without a placental diagnosis of DVM. Ultrasound measurements are shown in Table 3; other than a decreased umbilical PI at 30 weeks in the PGDM DVM group no other statistically significant difference was found between the groups.

There was no difference in mean HbA1c or fructosamine between pre-gestational diabetic mothers with or without placental DVM at any gestational age.

Regarding clinical outcome within the PGDM group itself, other than an increased birth weight in the DVM group (3911 $\mathrm{g}(+/-108 \mathrm{~g})$ vs. $3594 \mathrm{~g}(+/-57 \mathrm{~g}) \mathrm{p}<0.01)$ there was no other clinical outcomes different between those with a placental diagnosis of DVM and those without (Table 4).

In keeping with the absence of significant difference between mean birth weight between diabetic groups with and without DVM, there was also no difference in the birth weight centiles between the groups. Neither was there any difference between diabetic groups regarding perinatal outcome (where poor perinatal outcome was defined as Apgars at 5 min less than 7, cord pH less than 7.2 or admission to the unit for indication other than hypoglycaemia) (Table 4).

Table 2

Histological findings.

\begin{tabular}{llll}
\hline & $\begin{array}{l}\text { Non diabetic } \\
\text { normal controls } \\
(\mathrm{n}=77)\end{array}$ & $\begin{array}{l}\text { Pre-gestational } \\
\text { diabetes mellitus } \\
(\mathrm{n}=74)\end{array}$ & p value \\
\hline $\begin{array}{l}\text { 1. Cord } \\
\text { Single umbilical artery }\end{array}$ & $1 / 77(1.3 \%)$ & $2 / 74(2.7 \%)$ & NS \\
$\quad$ Funisitis & $2 / 77(2.6 \%)$ & $1 / 74(1.3 \%)$ & NS \\
2. Membranes & & & \\
Chorioamnioitis & $10 / 77(12.9 \%)$ & $5 / 74(6.7 \%)$ & \\
$\quad$ Meconium laden macrophages & $12 / 77(15.6 \%)$ & $4 / 74(5.4 \%)$ & \\
& & & \\
3. Parenchyma & & & NS \\
$\begin{array}{l}\text { Delayed villous maturation } \\
\text { Accelerated villous maturation } \\
\text { Chorangiosis }\end{array}$ & $4 / 77(5.2 \%)$ & $3 / 74(4.1 \%)$ & NS \\
$\begin{array}{l}\text { Villitis } \\
\text { Uteroplacental insufficiency }\end{array}$ & $2 / 77(2.6 \%)$ & $2 / 74(2.7 \%)$ & \\
\hline
\end{tabular}


Table 3

Third trimester ultrasound parameters in women with pre-gestational diabetes (PGDM) with and without a placental diagnosis of DVM.

\begin{tabular}{|c|c|c|c|}
\hline & $\begin{array}{l}\text { PGDM no DVM } \\
(\mathrm{n}=53)\end{array}$ & $\begin{array}{l}\text { PGDM with DVM } \\
(\mathrm{n}=21)\end{array}$ & $\mathrm{p}$ value \\
\hline \multicolumn{4}{|c|}{ Umbilical artery } \\
\hline \multicolumn{4}{|c|}{ Pulsatility index } \\
\hline - 30 weeks & $1.0(+/-0.03)$ & $0.88(+/-0.03)$ & 0.03 \\
\hline - 33 weeks & $0.95(+/-0.02)$ & $0.86(+/-0.03)$ & 0.11 \\
\hline - 36 weeks & $0.92(+/-0.02)$ & $0.85(+/-0.04)$ & 0.16 \\
\hline \multicolumn{4}{|c|}{$\begin{array}{l}\text { Liquor volume } \\
\text { (deepest cord free pool/cm) }\end{array}$} \\
\hline - 30 weeks & $6.79(+/-0.24)$ & $7.0(+/-0.44)$ & 0.66 \\
\hline - 33 weeks & $6.8(+/-0.24)$ & $10(+/-2.7)$ & 0.06 \\
\hline - 36 weeks & $7.32(+/-0.29)$ & $7.9(+/-0.61)$ & 0.3 \\
\hline \multicolumn{4}{|c|}{$\begin{array}{l}\text { Middle cerebral artery } \\
\text { pulsatility index }\end{array}$} \\
\hline - 30 weeks & $1.79(+/-0.06)$ & $1.79(+/-0.09)$ & 0.96 \\
\hline - 33 weeks & $1.71(+/-0.09)$ & $1.75(+/-0.09)$ & 0.73 \\
\hline - 36 weeks & $1.63(+/-0.05)$ & $1.55(+/-0.07)$ & 0.65 \\
\hline \multicolumn{4}{|l|}{ Ratio } \\
\hline \multicolumn{4}{|c|}{ UA PI: MCA PI } \\
\hline - 30 weeks & $0.59(+/-0.02)$ & $0.52(+/-0.03)$ & 0.07 \\
\hline - 33 weeks & $0.58(+/-0.02)$ & $0.51(+/-0.02)$ & 0.18 \\
\hline - 36 weeks & $0.57(+/-0.03)$ & $0.61(+/-0.05)$ & 0.08 \\
\hline
\end{tabular}

PGDM pregestational diabetes, umbilical artery (UA) pulsatility index, liquor volume (as $\mathrm{cm}$ depth of deepest cord free pool), middle cerebral artery (MCA) pulsatility index and ratio of UA PI to MCA PI are given as mean (+/-standard error) with $t$ test for comparison.

\section{Discussion}

DVM is linked with an increased risk of both intrauterine and neonatal deaths and may be part of the patho-physiology of increased perinatal mortality in diabetic pregnancies. In our population, pregestational diabetic women showed a significantly increased risk of DVM compared to non-diabetic controls. However the presence of placental DVM was not predicted by fetal ultrasound or by the clinical course of the pregnancy.

We assessed placental and fetal circulations and found a lower UA PI at 30 week gestation in the DVM group, with no difference evident at later gestation. Previous studies investigating UA PI in diabetic pregnancies have either shown increased UA PI with increasing glycaemia [23] or no difference [24]. Equally, increased UA SD ratio has been found in pregnancies of women with diabetic vasculopathy, and histological examination of the placenta has subsequently revealed fibrinoid necrosis, atherosis and thrombosis in the placental vessels [25]. It is unusual therefore that we have found a decrease in UA PI with a placental diagnosis of DVM in diabetics. This could suggest that DVM in pre-gestational diabetes is associated with a lower placental

\section{Table 4}

Clinical outcomes in infants of women with pre-gestational diabetes mellitus (PGDM) compared based on presence or absence of delayed villous maturation (DVM)

\begin{tabular}{|c|c|c|c|}
\hline & $\begin{array}{l}\text { PGDM no DVM } \\
(\mathrm{n}=53)\end{array}$ & $\begin{array}{l}\text { PGDM with DVM } \\
(\mathrm{n}=21)\end{array}$ & $\mathrm{p}$ value \\
\hline Length of diabetes (years) & $15(1-34)$ & $15(1-32)$ & 0.97 \\
\hline Birth weight (g) & $3594(+/-57)$ & $3911(+/-108)$ & 0.008 \\
\hline Birth weight centile & $91(15-99)$ & $97.5(25-99)$ & 0.13 \\
\hline $\begin{array}{l}\text { Birth weight greater } \\
\text { than 90th centile }\end{array}$ & $23 / 53$ & $7 / 20$ & 0.19 \\
\hline Apgars at $5 \mathrm{~min}$ & 9 & 9 & 0.68 \\
\hline \multicolumn{4}{|l|}{ Cord $\mathrm{pH}$} \\
\hline Umbilical artery & $7.26(+/-0.01)$ & $7.25(+/-0.02)$ & 0.7 \\
\hline Umbilical vein & $7.28(+/-0.01)$ & $7.27(+/-0.02)$ & 0.65 \\
\hline Admission to SCBU/NICU (\%) & $17 / 53(32 \%)$ & $8 / 21(38 \%)$ & 0.35 \\
\hline Poor perinatal outcome & $17 / 53$ & $3 / 21$ & 0.1 \\
\hline
\end{tabular}

vascular resistance, the exact aetiology of which remains to be further elucidated.

There is data to suggest that after 35 week gestation the middle cerebral artery could be more helpful in the assessment of fetal compromise rather than umbilical artery as the placenta has sufficient reserve to result in normal umbilical artery Doppler pulsatility index (PI) [26]. Typically the fetal middle cerebral artery PI is reduced in cases of fetal growth restriction [27] Though DVM is clinically different from uteroplacental insufficiency [10] we decided also to investigate middle cerebral artery PI as a possible predictor of fetal compromise with DVM. We also included the ratio of umbilical:MCA PI as a possible marker of fetal compromise. However no differences were noted in fetal middle cerebral artery circulation between those with and without placental DVM.

If maternal hyperglycaemia due to diabetes is associated with an increased risk of DVM then increasing hyperglycaemia with poorer maternal glycaemic control may increase this risk. However, there was no difference in HbA1c between women with DVM and those without at any gestational age, nor was there any difference in length of diabetes. The number of women with vascular disease secondary to diabetes was small (only 3 women were Whites Class R, and none were Class N) which does not allow for adequate comparison between groups. These results suggest that current measures of glycaemic control will not predict the development of DVM within the placenta.

A recent study from our group examining the stereological aspects of placentae of pre-gestational diabetic mothers noted that maternal glycaemia influenced capillary, but not stromal, development. This suggests that factors other than glycaemia have a role in placental development in pre-gestational diabetes [28].

This study was strengthened by the prospective recruitment of participants and the comparison with normal low risk non-diabetic pregnancies. Additionally we have prospectively collated detailed clinical and ultrasound parameters with which to compare to placental DVM. Finally, a multidisciplinary study involving both obstetricians and pathologists pooled knowledge of clinical conditions to form hypotheses, which could then be tested.

Though we have shown that diabetes nearly doubles the risk of DVM there is still a baseline risk of DVM of $14 \%$ in this low risk nondiabetic normal obstetric population. It is known that placental DVM is associated with increased intrauterine death in normally formed infants of non-diabetic mothers. A possible limitation of the study is that a number of placental DVM cases were at the milder end of the spectrum, with the potential for observer variation that may occur at the mild end of the spectrum of any diagnosis. However all placentae were assessed by one operator (EEM) thus reducing inter-observer bias and standardising the diagnosis of DVM. A more objective assessment of villous size and vascularity such as stereological assessment could overcome any potential bias in this microscopic diagnosis.

Delayed villous maturation remains an important placental finding in women with pre-gestational diabetes due to its known association with perinatal mortality. In a high risk-group such as women with PGDM it is clinically important to investigate possible clinical or ultrasound markers which may predict those at higher risk of developing perinatal morbidity or mortality. Despite this aim, we did not find any associated clinical markers on detailed analysis of antenatal ultrasound parameters nor perinatal outcome. Further studies are required to elucidate the clinical significance and ultrasound features of placental DVM in pre-gestational diabetic pregnancies.

\section{Funding}

The Health Research Board Ireland funded this research. The funding body had no role in the study design, data analysis nor writing of the manuscript. 


\section{Conflict of interest statement}

The authors have not conflict of interest.

\section{References}

[1] Confidential Enquiry into Maternal and Child Health. Pregnancy in women with Type 1 and Type 2 Diabetes 2002-2003. England, Wales and Northern Ireland. London: CEMACH; 2005. 80 pp.

[2] Evers IM, de Valk HW, Mol BWJ, ter Braak EWMT, Visser GHA. Macrosomia despite good glycaemic control in Type 1 diabetic pregnancy; results of a nationwide study in The Netherlands. Diabetologia 2002;45:1484-9.

[3] Russell NE, Holloway P, Quinn S, Foley M, Kelehan P, McAuliffe FM. Cardiomyopathy and cardiomegaly in stillborn infants of diabetic mothers. Pediatr Dev Pathol 2008;11(1):10-4.

[4] Russell N, Foley M, Kinsley B, Firth R, Coffey M, Mc Auliffe FM. Effects of pregestational diabetes on fetal cardiac function and structure. Am J Obstet Gynecol 2008;199(3):312-4.

[5] Russell NM, Higgins MF, Amouso M, Foley M, McAuliffe FM. Troponin T and pro-Btype natiuretic peptide in fetuses of type I diabetic mothers. Diabetes Care 2009;32(11):2050-5

[6] Sadlier TW. Fetal membranes and placenta. In: Sadlier TW, editor. Langman's medical embryology. Baltimore: Lippincott Williams and Wilkins; 2000. p. 136-57.

[7] Higgins MF, McAuliffe FM. A review of maternal and fetal growth factors in diabetic pregnancy. Curr Diabetes Rev 2010;6(2):116-25.

[8] Mooney EE, Doyle E, Gearhart P, Robboy SJ. Placenta-clinical scenarios. Pathology of the female reproductive tract. Elsevier; 2009. p. 863-80.

[9] Higgins M, McAuliffe FM, Mooney EE. Clinical associations with a placental diagnosis of delayed villous maturation: a retrospective study. Pediatr Dev Pathol Jul-Aug 2011;14(4):273-9.

[10] de Laat MW, van der Meij JJ, Visser GH, Franx A, Nikkels PG. Hypercoiling of the umbilical cord and placental maturation defect: associated pathology? Pediatr Dev Pathol 2007;10(4):292-9.

[11] Bernirschke K, Kaufmann P, Baergen R. Maternal diseases complicating pregnancy: diabetes, tumours, pre-eclampsia, lupus anticoagulant. In: Benirschke K, Kaufmann P, Baergen R, editors. Pathology of the Placenta. New York: Springer; 2006.

[12] Stallmach T, Hebisch G, Meier K, Dudenhausen J, Vogel M. Rescue by birth: defective placental maturation and late fetal mortality. Obstet Gynecol 2001;97(4): 505-9.

[13] Thomsen K, Lieschke G. Untersuchgen zure placenta-morphologie bei diabetes mellitus. Acta Endocrinol (Copenh) 1958;29:602-4.
[14] Vogel M. Olakopathia diabetic. Virchows Arch Pathol Anat Physiol Klin Med 1967;343:51-63.

[15] Fox H. Pathology of the placenta in maternal diabetes mellitus. Obstet Gynecol 1969;34:792-8.

[16] Singer D. The placenta in pregnancies complicated by diabetes mellitus. Perspect Pediatr Pathol 1984;8:199-212.

[17] Stoz F, Sxhuhmann RA, Schmid A. Morphometric investigations of terminal villi of diabetic placenta in relation to White classification of diabetes mellitus. J Perinat Med 1987;15(2):193-8.

[18] Evers IM, Nikkels PG, Sikkema JM, Visser GH. Placental pathology in women with Type 1 Diabetes and in a control group with normal and large-for-gestational-age infants. Placenta 2003;24:819-25.

[19] Laurini RN, Visser GHA, van Ballegooie E, Schoots CJF. Morphological findings in placentas of insulin-dependent diabetic patients treated with cutaneous subcutaneous insulin infusion (CSII). Placenta 1987;8:153-65.

[20] Madsen H, Ditzel J. Changes in red blood cell oxygen transport in diabetic pregnancy. Am J Obstet Gynecol 1982;143:421-4.

[21] Van Hoeven KH, Anyaegbunam A, Hochster H, Whitty JE, Distant J, Crawford C, et al. Clinical significance of increased histologic severity of acute inflammation of the fetal membranes and umbilical cord. Pediatr Pathol Lab Med 1996;16(5): 731-44.

[22] Knox WF, Fox H. Villitis of unknown aetiology: its incidence and significance in placentae from a British population. Placenta 1984;5(5):395-402.

[23] Bracero L, Figueroa R, Byrne DW, Han HJ. Comparison of umbilical Doppler velocimetry, NST and biophysical profile in pregnancies complicated by diabetes. J Ultrasound Med 1996;15:301-8.

[24] Landon MB, Gabbe SG, Bruner JP, Ludmir J. Doppler umbilical artery velocimetry in pregnancy complicated by insulin dependent diabetes mellitus. Obstet Gynecol 1989;73:961-5.

[25] Reece EA, Hagay Z, Assunakoupoulos E, Moroder W, Gabierilli S, DeGennaro N, et al. Diabetes mellitus in pregnancy and the assessment of umbilical artery waveforms using pulsed Doppler ultrasonography. J Ultrasound Med Feb 1994;13(2):73-80.

[26] Ong K, Kratzsch J, Kiess W, Costello M, Scott C, Dunger D. Size at birth and cord blood levels of insulin, insulin like growth factor I (IGF-I), IGF-II, IGF-binding protein 1, (IGFBP-1), IGFBP-3, and the soluble IGF-II/mannose-6-phosphate receptor in term human infants. The ALSPAC Study team. AVON Longitudinal Study of Pregnancy and Childhood. J Clin Endocrinol Metab 2000;85:4266-9.

[27] Langer O. Is normoglycaemia the correct threshold to prevent complications in the pregnant diabetic patient? Diabetes Rev 1996;4:2-10.

[28] Higgins M, Felle P, Mooney EE, Bannigan J, McAuliffe F. Stereology of the placenta in Type 1 and Type 2 Diabetes. Placenta Aug 2011;32(8):564-9. 